\title{
Prognostic relevance of increased Rac GTPase expression in prostate carcinomas
}

\author{
R Engers, S Ziegler, M Mueller ${ }^{1}$, A Walter, $R$ Willers $^{2}$ and H E Gabbert \\ Institute of Pathology, Heinrich-Heine-University, Moorenstr 5, 40225 Duesseldorf, Germany \\ ${ }^{1}$ Department of Urology, Heinrich-Heine-University, Duesseldorf, Germany \\ ${ }^{2}$ Department of Computational Statistics, Heinrich-Heine-University, Duesseldorf, Germany \\ (Requests for offprints should be addressed to R Engers; Email: engers@med.uni-duesseldorf.de)
}

\begin{abstract}
Rac proteins of the Rho-like GTPase family, including the ubiquitous Rac1, the hematopoiesisspecific Rac2, and the least-characterized Rac3 play a major role in oncogenic transformation, tumor invasion and metastasis. However, the prognostic relevance of Rac expression in human tumors has not been investigated yet. In the present study, Rac protein expression was analyzed in benign secretory epithelium, high-grade prostatic intraepithelium neoplasia (HG-PIN), and prostate carcinomas of 60 R0-resected radical prostatectomy specimens by semiquantitative immunohistochemistry. Thus, Rac proteins were significantly strongly expressed in HG-PIN $(P<0.001)$ and prostate carcinomas $(P<0.001)$ when compared with benign secretory epithelium. Accordingly, all tumor tissues analyzed by isoform-specific real-time PCR $(n=7)$ exhibited significantly higher RNA expression levels of Rac (i.e. sum of Rac1 and Rac3 expression levels) than the respective benign counterparts $(P=0.018)$ and this appeared to result mainly from increased expression of the Rac3 isoform as verified by immunoblotting. Univariate analyses showed statistically significant associations of increased Rac protein expression in prostate cancer $(P=0.045)$, preoperative prostate-specific antigen levels $(P=0.044)$, pT stage $(P=0.002)$, and Gleason score $(P=0.001)$ with decreased disease-free survival (DFS). This prognostic effect of increased protein expression of Rac remained significant even in a multivariate analysis including all these four factors (relative risk $=3.22,95 \%$ confidence interval $=1.04-10.00$; $P=0.043$ ). In conclusion, our data suggest that increased Rac protein expression in prostate cancer relative to the corresponding benign secretory epithelium is an independent predictor of decreased DFS and appears to result mainly from increased expression of the Rac3 isoform.
\end{abstract}

Endocrine-Related Cancer (2007) 14 245-256

\section{Introduction}

Prostate cancer is the most common malignant tumor type in men and the second leading cause of cancer death in men in North America and Western Europe. In 2006, prostate cancer alone was expected to account for approximately $33 \%$ of all newly diagnosed cancer cases in men (Jemal et al. 2006). Of these, however, only a fraction would lead to cancer-related death if left untreated (Nelson et al. 2003, Jemal et al. 2006). Consequently, there is a great need for markers, which accurately predict the risk of disease progression in patients with prostate cancer and thus allow appropriate treatment planning. At present, several clinical parameters including tumor stage, Gleason score (GS), and the serum level of preoperative prostate-specific antigen (PSA) are typically used to assess the risk of disease progression at the time of diagnosis (Partin et al. 1997). Unfortunately, these parameters are still not sufficiently precise to allow optimal treatment planning, as recently reviewed by Ross et al. (2003).

Rac proteins are members of the Rho family of small GTPases involved in the regulation of actin dynamics. So far, three highly homologous Rac proteins have been described in mammals: the ubiquitous Rac1, the hematopoiesis-specific Rac2, and the least-characterized Rac3 (for review see: Wennerberg \& Der 2004). Rac1 is a major regulator of the actin cytoskeleton (Ridley et al. 1992), which affects cell cycle progression (Olson et al. 1995), cell adhesion (Hordijk et al. 1997, Kuroda et al. 1998), 
migration (Keely et al. 1997, Sander et al. 1998, Engers et al. 2001), invasion (Hordijk et al. 1997, Keely et al. 1997, Engers et al. 2001, 2006b), and metastasis (Shan et al. 2005) of tumor cells. Moreover, Rac1 has been implicated in oncogenic transformation of cells. Thus, Rac1 is essential for Ras- and Src-induced transformation of NIH3T3 cells (Qiu et al. 1995, Servitja et al. 2003) and in line with this, mice deficient in the Rac-specific activator, Tiam1, are resistant to the development of Rasinduced skin tumors (Malliri et al. 2002). In addition, Rac1 is also required for BCR/ABL-mediated leukemogenesis (Skorski et al. 1998). In contrast to Rac1, Rac2 is specifically expressed in cells of hematopoietic origin (Didsbury et al. 1989, Wennerberg \& Der 2004) and mice genetically deficient in Rac2 are characterized by defects in cellular functions of diverse hematopoietic cells (Croker et al. 2002a,b, Gu et al. 2002, 2003, Carstanjen et al. 2005). Rac3 is most highly expressed in brain, but has also been found in other organs including placenta, kidney, pancreas, and heart (Haataja et al. 1997). However, Rac3 expression in human prostate and prostate carcinomas has not been reported yet. Unlike Rac1 knockout animals, which do not survive embryogenesis (Sugihara et al. 1998), Rac3 knockout animals do survive and show no obvious developmental defects (Corbetta et al. 2005). Nevertheless, Rac3 is hyperactive in breast cancers (Mira et al. 2000) and recently, like Rac1, it has been implicated in the regulation of migration and invasion of ganglioblastoma and breast carcinoma cells (Chan et al. 2005). Moreover, activation of Rac3 has recently been shown to cause transformation of NIH3T3 cells (Joyce \& Cox 2003).

Despite these functions of Rac proteins, the potential prognostic relevance of Rac expression in human tumors has not been investigated yet. In the present study, we have analyzed Rac protein expression in benign secretory epithelium, high-grade prostatic intraepithelium neoplasia (HG-PIN), and prostate carcinomas by semiquantitative immunohistochemistry. We found that Rac protein expression is significantly higher in prostate carcinomas and HG-PIN lesions than in the corresponding benign secretory epithelium and we provide evidence that this results mainly from increased expression of the Rac3 isoform. Moreover, in multivariate analysis, Rac overexpression in prostate cancer relative to the corresponding benign secretory epithelium proved to be an independent predictor of decreased disease-free survival (DFS) for patients with prostate cancer.

\section{Materials and methods}

\section{Patients}

In the present study, we investigated Rac protein expression by semiquantitative immunohistochemistry in 60 patients with R0-resected prostate cancer, who underwent radical prostatectomy at the University Hospital of Duesseldorf. Median patient age was 67 years (range, 55-79 years). Patients were excluded from the study if they had received neoadjuvant hormonal therapy, if surgical margins were positive, and/or if postoperative PSA values remained above $0.3 \mathrm{ng} / \mathrm{ml}$. Disease recurrence was considered in the following circumstances: a) PSA measurement above $0.3 \mathrm{ng} / \mathrm{ml}$; b) radiological or histological evidence of local recurrence or metastasis. Mean preoperative PSA level was $14.4 \pm 12 \mathrm{ng} / \mathrm{ml}$ (range, $1.2-60.5 \mathrm{ng} / \mathrm{ml}$ ). For one patient the preoperative PSA level was unknown. Appropriate follow-up data were available for 53 patients. Of these, 1 patient died from prostate cancer, 7 from other diseases, and 36 were censored. Median follow-up time of survivors was 7.2 years (range, 214.4 years). The study and its execution were approved by the ethics committee of the Heinrich-HeineUniversity of Duesseldorf.

\section{Testing of the monoclonal Rac antibody}

To verify whether or not the Rac-specific monoclonal antibody we aimed to use for immunohistochemistry (BD Biosciences, Heidelberg, Germany; Clone 102) recognizes all Rac isoforms, which may be expressed by epithelial cells (i.e. Rac1 and Rac3), Cos7 cells were transiently transfected with empty vector (pMT2SM), wild type Rac1, or wild type Rac3 (each kindly provided by Dr J G Collard, Amsterdam, The Netherlands) by the DEAE-Dextran method. The cells were washed twice in PBS (Sigma) and subsequently incubated in a solution containing $2 \mu \mathrm{g}$ of the respective plasmid and $500 \mu \mathrm{g}$ DEAE-Dextran (Sigma) for $20 \mathrm{~min}$ at $37^{\circ} \mathrm{C}$. Then, chloroquine ( $80 \mu \mathrm{M}$; Sigma) was added and cells were incubated for $2.5 \mathrm{~h}$ at $37^{\circ} \mathrm{C}$. Supernatants were subsequently removed and cells were incubated for $2.5 \mathrm{~min}$ in $10 \%$ DMSO at room temperature. Afterwards, supernatants were replaced by normal growth medium (Dulbecco's modified Eagle medium) supplemented with $10 \%$ fetal calf serum and antibiotics (all Sigma) and cells were incubated at $37^{\circ} \mathrm{C}$. Two days after transfection, cells were lysed and Rac protein expression was analyzed by imunoblotting as previously described (Engers et al. 2006b). Equal protein loading was confirmed by re-incubating the same membranes with a mouse 
monoclonal antibody against glyceraldehyde-3-phosphate dehydrogenase (GAPDH; Abcam, Bad Nauheim, Germany; dilution 1:5000).

\section{Pathological and immunohistochemical evaluation}

All tumors were staged using the tumor-nodemetastasis (TNM) system (Sobin \& Wittekind 2002) and graded according to the system described by Gleason (1988). In addition, all tumors were evaluated for blood vessel invasion (BVI), lymph vessel invasion (LVI), perineural invasion (PNI), and the presence of HG-PIN by the study pathologist. For immunohistochemistry representative $5 \mu \mathrm{m}$ sections of paraffinembedded tissue specimens were incubated with a Rac-specific monoclonal antibody (BD Biosciences; Clone 102; dilution 1:250), using a Ventana BenchMark immunohistochemical autostainer (Ventana Medical Systems, Munich, Germany) according to the manufacturer's instructions. As a negative control, immunostaining of representative sections were performed by omitting the primary antibody. For quantification, the percentage of positive cells was divided into five groups: $0,0 \% ; 1,1-10 \% ; 2,11-50 \%$; $3,51-80 \%$; and $4,>80 \%$. A scale from 0 (no staining) to 3 (strong immunoreactivity) was assigned to staining intensity. Immunoreactive scores were calculated by multiplying percentage score of positive cells times staining intensity score, yielding an end score of 0-12 (Engers et al. 2006a). This scoring system was used in the present study, because it is well established in routine diagnostic pathology. For instance, in breast cancer, this scoring system is used to determine hormone receptor expression semiquantitatively and based on the scores provided by the pathologist, clinicians decide whether or not patients will be treated with distinct drugs. In order to exclude potential influences of unknown differences in tissue fixation on immunohistochemical results, Rac immunoreactive ratios, defined as Rac immunoreactive scores in prostate cancer or HG-PIN respectively, relative to Rac immunoreactive scores in the corresponding benign secretory epithelium in the same section, were used for statistical analyses rather than absolute Rac immunoreactive scores. These ratios could be calculated, since in all cases analyzed immunoreactive scores in benign secretory epithelial cells were $\geq 1$. Rac immunoreactive ratios $>1$ were defined as Rac overexpression. Clinical data collection and immunohistochemical analyses were performed independently of each other, with respective investigators blinded to the clinical outcome and Rac result until the study was completed.

\section{Tissue collection}

To verify whether increased Rac protein expression in prostate cancer as observed by immunohistochemistry is also detectable on the RNA level and to identify the Rac isoforms involved, representative samples of prostate cancer and the respective benign counterparts were collected. Since frozen material of the patients included in our immunohistochemical study was not available, representative tissue samples were collected from seven successively operated radical prostatectomy specimens within $15 \mathrm{~min}$ after surgery by the study pathologist. Normal and tumor tissues were identified by gross morphology and immediately verified by microscopy on frozen sections. The percentage of tumor in the respective samples was at least $60 \%$. Collected and microscopically verified tissue samples were snap-frozen in liquid nitrogen and stored at $-80{ }^{\circ} \mathrm{C}$ until further processing.

\section{RNA extraction and real-time PCR}

Frozen tissues were homogenized by mortar and pestle and total RNA was isolated using the RNeasy Mini Kit (Qiagen) including on-column DNA digestion with RNase-free DNase (Qiagen). RNA was reverse transcribed using oligo-dT and Omniscript Reverse Transcriptase (Qiagen). The quantitative real-time PCR was performed using the LightCycler instrument (Roche) and Platinum SYBR Green qPCR SuperMixUDG (Invitrogen) in total volume of $20 \mu \mathrm{l}$. The generation of specific PCR products was confirmed by melting curve analysis and gel electrophoresis and sequencing. Primer efficiencies of each primer pair were determined by means of a logarithmic dilution of a cDNA derived from the prostate carcinoma cell line DU145 and a representative cDNA mix respectively. The following formula was used to calculate the respective primer pair efficiency: $E=10^{(-1 / \text { slope })}$ (crossing point plotted versus log of template concentration). Linear standard curves were generated with a logarithmic dilution of cDNA mix by using the LightCycler3 software (Roche). All samples were normalized to the level of GAPDH. To analyze the expressions of Rac1, Rac2, Rac3, and GAPDH respectively, the following primers were used

GAPDH forward: 5'-CATGTTCCAATATGATTCCACC- $3^{\prime}$

GAPDH reverse: 5'-GATGGGATTTCCATTGATGAC- $3^{\prime}$ 
Rac1 forward: 5'-AACCAATGCATTTCCTGGAG-3' Rac1 reverse: $5^{\prime}$-CAGATTCACCGGTTTTCCAT-3' Rac2 forward: $5^{\prime}$-CATCAGCTACACCACCAACG- $3^{\prime}$ Rac2 reverse: 5'-TTGCTGTCCACCATCACATT-3' Rac3 forward: $5^{\prime}$-GGGAAGACATGCTTGCTGAT- $3^{\prime}$ Rac3 reverse: $5^{\prime}$ - CCATCACGTTGGCAGAGTAG-3'

\section{Protein extraction and Rac3 immunoblotting}

Frozen tissues were homogenized by mortar and pestle and lysed in a buffer containing $150 \mathrm{mM} \mathrm{NaCl}, 20 \mathrm{mM}$ Tris, $\mathrm{pH} 7.5,1 \%$ Triton-X 100, and protease inhibitors (Roche). Protein concentrations were determined by the Bradford method and equal amounts of protein were separated by SDS-PAGE. Expression levels of Rac3 were determined by immunoblotting using a Rac3-specific rabbit polyclonal antibody, kindly provided by Dr I de Curtis (Milano, Italy; Bolis et al. 2003). Equal protein loading was confirmed by re-incubating the same membranes with a mouse monoclonal antibody against GAPDH (Abcam; dilution 1:5000). For detection, the enhanced chemiluminescence detection system was used.

\section{Statistics}

Rac protein expression levels in benign, preneoplastic (HG-PIN), and neoplastic lesions as determined by semiquantitative immunohistochemistry as well as RNA expression levels of Rac isoforms in prostate cancer and the corresponding normal counterparts were compared by the Wilcoxon test. Associations between Rac overexpression and different clinicopathological parameters were assessed with Fisher's exact test. DFS was calculated from the date of radical prostatectomy to the date of recurrence or last followup. The data on various biochemical and pathological parameters as well as Rac expression were analyzed by Cox proportional hazard method, using univariate and multivariate analysis. All $P$ values were two-sided. The analyses were performed with SPSS statistical software version 12.0 (SPSS GmbH, Munich, Germany).

\section{Results}

\section{Patients}

Based on histological evaluation, 37 tumors were categorized as pT2 (organ-confined tumors; 62\%) and 23 tumors as pT3 (tumors with extraprostatic extension; $38 \%$ ). In one patient (2\%) a lymph node micrometastasis was observed. No distant metastases were detected at the time of radical prostatectomy. GSs ranged from
5 to 10 with GS 5 in 2 patients (3.3\%), GS 6 in 9 patients (15\%), GS 7 in 38 patients (63.3\%), GS 8 in 4 patients (6.7\%), GS 9 in 6 patients (10\%), and GS 10 in 1 patient (1.7\%). PNI was observed in 46 patients (77\%), LVI in 6 patients (10\%), and BVI in 2 patients $(3 \%)$.

\section{The Rac-specific antibody recognizes Rac1 and Rac3 isoforms}

To our best knowledge, all Rac-specific antibodies suitable for immunohistochemistry on paraffinembedded material exhibit at least some crossreactivity among the different Rac isoforms, because of a high sequence identity of $\approx 88 \%$ amongst these isoforms (Wennerberg \& Der 2004). According to the manufacturer's information, the monoclonal Racspecific antibody we aimed to use for our immunohistochemical study does not react with Rac-related Rho and $\mathrm{Cdc} 42$ proteins. However, its cross-reactivity among different Rac isoforms has not been tested yet. Therefore, we first investigated whether this antibody recognizes all Rac isoforms, which may be expressed by epithelial cells (i.e. Rac1 and Rac3). By transient overexpression of the respective cDNAs in Cos7 cells and immunoblotting, we could demonstrate that this antibody recognizes Rac1 and Rac3 proteins and that both proteins are of the same molecular weight (Fig. 1). From this we conclude that Rac protein expression in epithelial cells as determined by means of this antibody reflects expression of both Rac1 and Rac3 isoforms.

\section{Rac protein expression in prostate cancer, HG-PIN lesions, and benign prostate tissues as determined by semiquantitative immunohistochemistry}

By immunohistochemistry, we found that in human prostate tissues Rac was expressed by benign epithelial cells, HG-PIN lesions, prostate cancer, and inflammatory cells (Fig. 2A and B). In contrast, fibromuscular stromal cells were almost entirely negative (Fig. 2B). Interestingly, in inflammatory cells, benign secretory epithelial cells, and HG-PIN lesions Rac expression were exclusively restricted to the cytoplasm (Fig. 2A and $\mathrm{B}$ ), whereas in a small proportion of prostate cancers $(n=7,11.7 \%)$, some of the tumor cells exhibited an additional nuclear expression pattern (Fig. 2C). Moreover, while Rac expression in inflammatory cells, including lymphocytes, plasma cells, neutrophilic granulocytes, and histiocytes was constantly very strong, Rac expression in prostate cancer, HG-PIN lesions, and the respective benign prostatic glands sometimes varied considerably. 


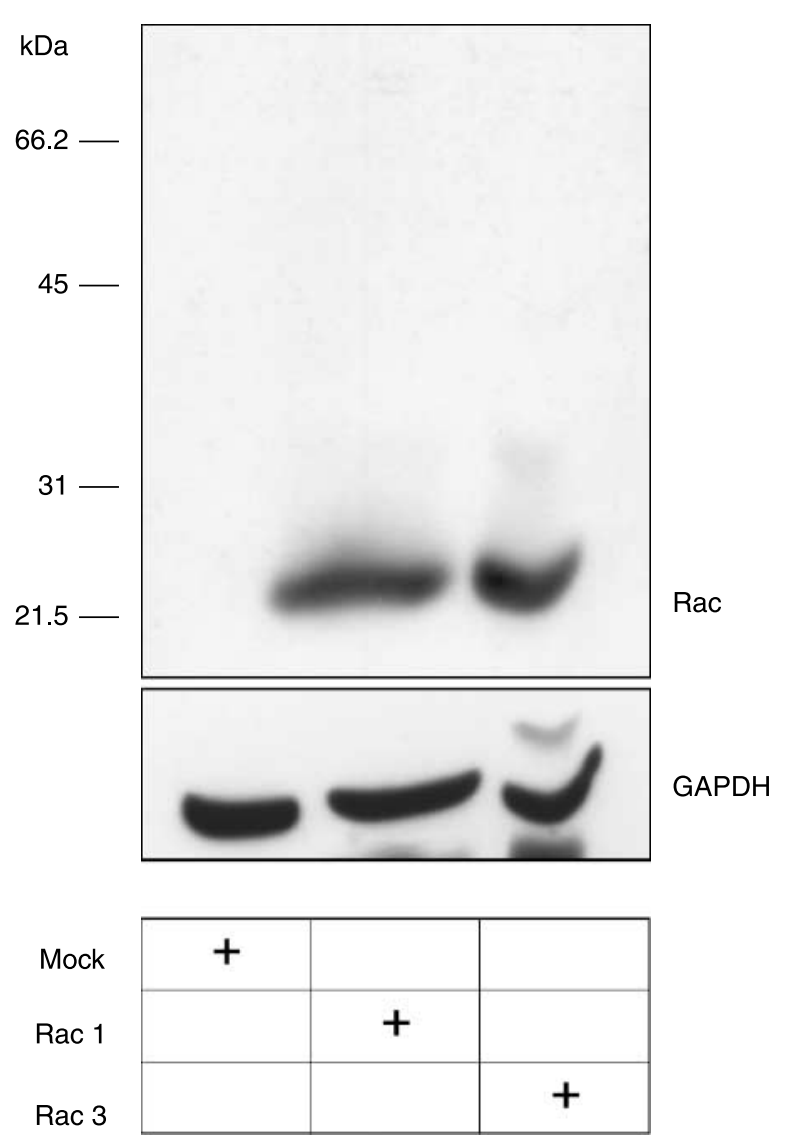

Figure 1 Rac antibody testing by immunoblotting. To investigate, whether the monoclonal Rac-specific antibody recognizes all Rac isoforms, which may be expressed by epithelial cells (e.g., Rac1 and Rac3), Cos7 cells were transiently transfected with empty vector (mock), Rac1, and Rac3 respectively. Subsequent immunoblotting revealed that the antibody recognizes both Rac1 and Rac3 proteins and that both proteins are of about the same molecular weight. Equal protein loading was verified by re-incubating the membranes with an antibody against GAPDH. Results are representative for three independent experiments.

Therefore, Rac expression in benign, premalignant, and malignant epithelial cells was analyzed by semiquantitative immunohistochemistry. We found that in 30 out of 60 carcinomas $(50 \%)$ Rac expression levels were higher, in 27 carcinomas $(45 \%)$ identical to, and in 3 carcinomas (5\%) lower than in the respective corresponding benign secretory epithelial cells. Moreover, in 27 out of 53 HG-PIN lesions (51\%) Rac expression levels were higher, in 22 HG-PIN lesions (41.5\%) identical to, and in 4 HG-PIN lesions $(7.5 \%)$ lower than in the corresponding benign secretory epithelial cells. In total, Rac expression levels proved significantly higher in prostate carcinomas $(P<0.001)$ and HG-PIN lesions $(P<0.001)$ than in the respective benign secretory epithelial cells (Fig. 2D). However, no difference was seen between Rac expression levels in prostate carcinomas and HG-PIN lesions.

\section{Evaluating potential associations between Rac protein expression in prostate cancer and different clinicopathological factors}

To analyze potential associations between Rac protein expression in prostate cancer and different clinicopathological factors (age, preoperative PSA, LVI, BVI, PNI, pT, pN, GS, and disease recurrence), Rac immunoreactive ratios (e.g., Rac expression levels in prostate cancer relative to those in the corresponding benign secretory epithelial cells) were dichotomized into two categories ( $>1$ vs $\leq 1$, e.g., Rac overexpression versus no overexpression of Rac). Given a mean preoperative PSA level of $14.4 \mathrm{ng} / \mathrm{ml}$ in our cohort, preoperative PSA levels were dichotomized into $<15 \mathrm{ng} / \mathrm{ml}$ vs $\geq 15 \mathrm{ng} / \mathrm{ml}$. GS was subdivided into $<7$ vs $\geq 7$, and pT stage into organconfined tumors (pT2) versus tumors with extraprostatic extension (pT3). Other parameters such as LVI, BVI, PNI, and $\mathrm{pN}$ were categorized into present versus not present. As shown in Table 1, Rac overexpression in prostate carcinoma was statistically significantly associated with the presence of PNI $(P=0.005)$, high GSs (i.e. $\geq 7 ; P=0.043)$, and with disease recurrence $(P=0.041)$, but not with any other parameter.

\section{Evaluating the prognostic relevance of increased Rac protein expression and different clinico- pathological factors in prostate cancer}

Since the follow-up times of patients in this cohort varied between 24 and 173 months, the Cox proportional hazards model and single-parameter analysis was used to determine the prognostic significance of each of the different clinicopathological factors (i.e. age, preoperative PSA, LVI, BVI, PNI, pT, $\mathrm{pN}$, and GS) as well as Rac protein overexpression. As shown in Table 2, age, LVI, BVI, PNI, and pN did not significantly predict DFS. In contrast, Rac protein overexpression $(P=0.045)$, preoperative PSA level $(P=0.044)$, pT stage $(P=0.002)$, and GS $(P=0.001)$ significantly predicted decreased DFS (Table 2$)$.

Most importantly, the association of Rac overexpression with decreased DFS remained significant even in a multivariate analysis, including all these four factors (relative risk $=3.22,95 \%$ confidence interval $=$ 1.04-10.00; two-sided $\chi^{2} P=0.043$; Table 3). The fact, as to whether nuclear Rac expression was present in at least some of the tumor cells or not, proved to be of no 

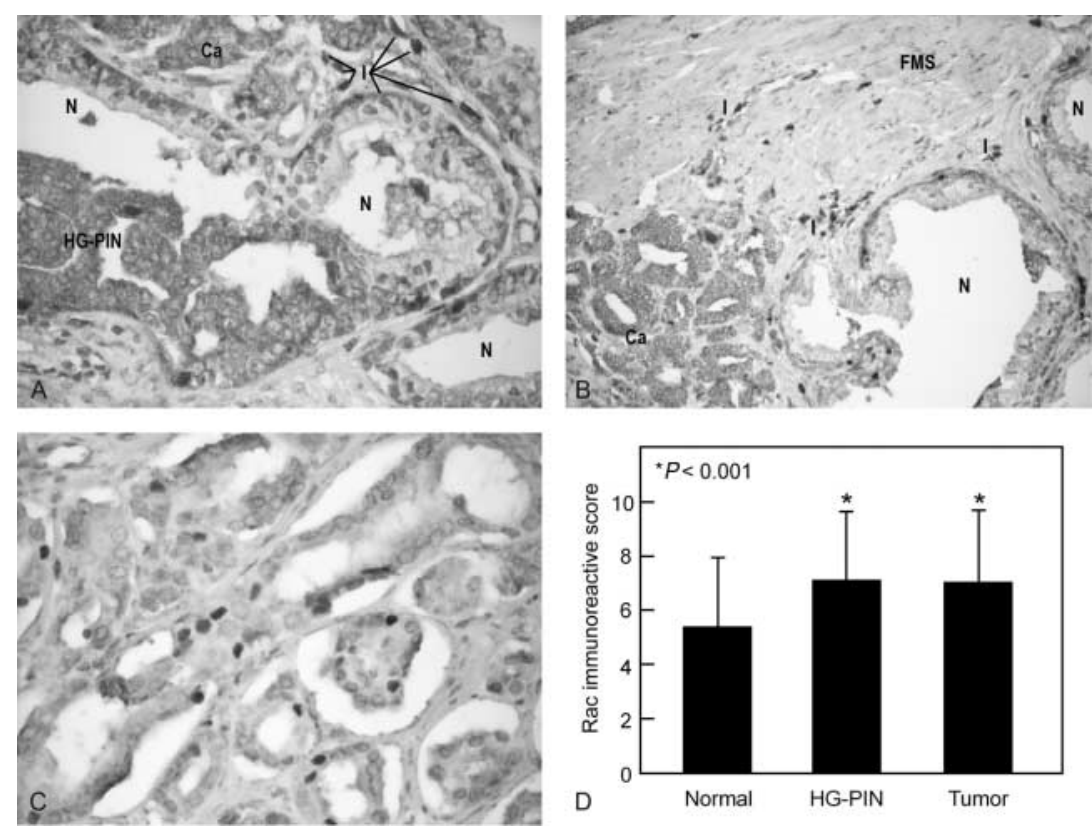

Figure 2 Rac protein expression in human prostate cancer as determined by immunohistochemistry. Rac expression was strongly increased in preneoplastic HG-PIN lesions (A) and prostate cancer (Ca) (B) as compared with adjacent normal secretory epithelium (N). Moreover, Rac was strongly expressed by inflammatory cells (I), whereas fibromuscular stromal cells (FMS) were almost entirely negative. (C) Nuclear expression of Rac as found in a small proportion of prostate cancer cells. (D) Comparison of Rac expression levels (mean \pm s.D.) in benign secretory epithelium $(n=60)$, HG-PIN $(n=53)$, and prostate cancer $(n=60)$ by semiquantitative immunohistochemistry. Rac expression levels were significantly higher in prostate cancer and HG-PIN than in benign secretory epithelium (two-sided Wilcoxon test). Original magnifications: $(A) \times 400,(B) \times 200,(C), \times 400$.

statistical relevance in neither terms of an association between Rac protein overexpression and different clinicopathological features, nor in terms of an association between Rac protein overexpression and decreased DFS.

\section{Evaluating Rac expression in prostate cancer and its benign counterpart on the RNA level by real-time PCR}

To verify whether increased Rac expression in prostate cancer as observed on the protein level by immunohistochemistry was also detectable on the RNA level, seven representative samples of prostate cancer and the respective normal counterparts were analyzed by real-time PCR. These analyses were initially performed for Rac1 and Rac3 only, because in contrast to Rac1 and Rac3, Rac2 is known to be exclusively expressed by hematopoietic cells (Wennerberg \& Der 2004). Thus, we found that the sum of Rac1 and Rac3 expression levels was significantly higher in prostate carcinomas $($ mean $=1.65$-fold, range 1.11 - to 2.61 fold, $P=0.018$ ) than in the corresponding normal tissues (Fig. 3A). More specifically, in six out of seven cases RNA expression levels of Rac1 were higher
$($ mean $=1.39$-fold, range $1.04-$ to 2.23 -fold $)$ in prostate cancer than in the corresponding normal tissue, whereas in one case we observed a marked downregulation of Rac1 in prostate cancer by $\sim 50 \%$ in comparison with the normal counterpart (Fig. 3B). In total, however, these differences in Rac1 RNA expression levels between tumor and benign tissue samples were not sufficient to reach statistical significance $(P=0.176)$.

Since Rac1 is known to be also expressed by inflammatory cells, differences in Rac1 expression between prostate cancer samples and the respective normal tissues as detected by real-time PCR might result from differences in the extent of inflammation. To clarify this, the extent of inflammation in our samples was analyzed both by histological examination of the respective frozen tissue sections and by investigating expression of the hematopoietic cell-specific Rac2 isoform by real-time PCR. Histological examination showed only minimal to mild inflammation in all tissue samples and in all cases the extent of inflammation in tumor samples was either similar to or even weaker than in the respective normal counterparts (data not shown). Accordingly, mRNA expression levels of Rac2 were found to be reduced rather than increased in tumor 
Table 1 Clinicopathologic features of Rac overexpression in prostate cancer ${ }^{\mathrm{a}}$

\begin{tabular}{|c|c|c|c|}
\hline Factor & Rac overexpression no $(n=26)$ no. $(\%)$ & Rac overexpression yes $(n=34)$ no. $(\%)$ & $P$ value $^{\mathrm{b}}$ \\
\hline \multicolumn{4}{|l|}{ Age, y } \\
\hline$<65$ & $12(20)$ & $14(23)$ & 0.603 \\
\hline$\geq 65$ & $19(32)$ & $15(25)$ & \\
\hline \multicolumn{4}{|c|}{ Preoperative $\mathrm{PSA}^{\mathrm{c}}, \mathrm{ng} / \mathrm{ml}$} \\
\hline$<15$ & $21(36)$ & $16(27)$ & 0.432 \\
\hline$\geq 15$ & $10(17)$ & $12(20)$ & \\
\hline \multicolumn{4}{|c|}{ Disease recurrence ${ }^{d}$} \\
\hline No & $24(45)$ & $12(23)$ & $0.041^{*}$ \\
\hline Yes & $6(11)$ & $11(21)$ & \\
\hline \multicolumn{4}{|l|}{ LVI } \\
\hline No & $28(47)$ & $26(43)$ & 1.0 \\
\hline Yes & $3(5)$ & $3(5)$ & \\
\hline \multicolumn{4}{|l|}{ BVI } \\
\hline No & $29(48)$ & $29(48)$ & 0.492 \\
\hline Yes & $2(3)$ & $0(0)$ & \\
\hline \multicolumn{4}{|l|}{ PNI } \\
\hline No & 12 (20) & 2 (3) & $0.005^{\star}$ \\
\hline Yes & 19 (32) & $27(45)$ & \\
\hline \multicolumn{4}{|l|}{ pT } \\
\hline pT2 & $22(37)$ & $15(25)$ & 0.184 \\
\hline рT3 & $9(15)$ & $14(23)$ & \\
\hline \multicolumn{4}{|l|}{$\mathrm{pN}$} \\
\hline pNO & $31(52)$ & $28(47)$ & 0.483 \\
\hline pN1 & $0(0)$ & $1(2)$ & \\
\hline \multicolumn{4}{|l|}{ GS } \\
\hline$<7$ & 9 (15) & 22 (37) & $0.043^{\star}$ \\
\hline$\geq 7$ & $2(3)$ & $27(45)$ & \\
\hline
\end{tabular}

${ }^{\mathrm{a}}$ Because of rounding percentages may not equal $100 \%$.

${ }^{\mathrm{b}}$ Fisher's exact test, two-sided.

'Since preoperative PSA was a continuous estimate with the mean PSA level for the entire cohort of patients (PSA levels were available for 59 out of 60 patients) as $14.4 \mathrm{ng} / \mathrm{ml}$, we divided the cohort into those with PSA levels $<15$ and $\geq 15$.

${ }^{\mathrm{d}}$ Appropriate follow-up was available for 53 out of 60 patients.

*Statistically significant. LVI, lymph vessel invasion; BVI, blood vessel invasion; PNI, perineural invasion; GS, Gleason score.

specimens as compared with the respective normal tissue samples (Fig. 3C). This indicates that the tendency of increased Rac1 expression in tumor samples as detected by real-time PCR can indeed be attributed to the tumor cell compartment rather than being caused by inflammatory cells.
In contrast to Rac1, all prostate cancer samples examined exhibited higher RNA expression levels of Rac3 than the respective benign counterparts (mean $=$ 3.10 -fold, range 1.43 - to 4.80 -fold) and these differences proved to be statistically significant $(P=0.018$; Fig. 3D). These differences cannot simply

Table 2 Univariate analysis of pre- and postoperative parameters as well as Rac overexpression

\begin{tabular}{lcccc}
\hline Parameter & $\chi^{2}$ & $\boldsymbol{P}$-value & Hazard ratio & 95\% Cl \\
\hline Age & 1.658 & 0.202 & 1.058 & $0.970-1.155$ \\
Preoperative PSA & 4.174 & $0.044^{*}$ & 1.035 & $1.001-1.070$ \\
LVI & 2.323 & 0.142 & 2.604 & $0.725-9.346$ \\
BVI & 0.592 & 0.612 & 21.639 & $0.000-3126101$ \\
PNI & 2.105 & 0.164 & 2.907 & $0.646-13.158$ \\
PT & 9.451 & $0.006^{*}$ & 5.805 & $1.657-20.339$ \\
pN & 3.726 & 0.091 & 6.098 & $0.750-50$ \\
GS & 12.685 & $0.001^{*}$ & 2.121 & $1.369-3.286$ \\
Rac overexpression & 4.344 & $0.045^{*}$ & 2.793 & $1.021-7.634$ \\
\hline
\end{tabular}

$\mathrm{Cl}$, confidence interval. Cox proportional hazard model and single parameter analysis were used to determine the prognostic significance of age, preoperative PSA, LVI +/-, BVI +/-, PNI +/-, pT stage, pN stage, GS, and Rac overexpression +/-. Age, preoperative PSA, and GS were used as continuous variables.

*Statistically significant. 
Table 3 Multivariate analysis of Rac overexpression with disease-free survival in patients with prostate cancer by the Cox proportional hazard method

\begin{tabular}{lcc}
\hline Variable & $\begin{array}{c}\boldsymbol{P} \\
\text { value }^{\mathrm{a}}\end{array}$ & HR $(95 \% \mathrm{Cl})^{\mathrm{a}}$ \\
\hline $\begin{array}{l}\text { Rac overexpression (yes } \\
\quad \text { versus no) }\end{array}$ & 0.043 & $3.22(1.04-10.00)$ \\
$\begin{array}{l}\text { pT (pT3 versus pT2) } \\
\text { Gleason score } \\
\text { preoperative PSA }\end{array}$ & 0.042 & $3.94(1.05-14.71)$ \\
\hline
\end{tabular}

$\mathrm{HR}$, hazard ratio; $\mathrm{Cl}$, confidence interval.

${ }^{a}$ All statistical tests were two-sided.

be attributed to the choice of a distinct housekeeping gene (i.e. GAPDH) for normalization, because normalization for another housekeeping gene (adenine phosphoribosyltransferease) gave rise to similar results (data nor shown). Interestingly, all carcinomas exhibited higher ratios of Rac3/Rac1 RNA expression (mean $=0.77$, range $0.10-1.80$ ) than the respective normal counterparts $($ mean $=0.26$, range $0.07-0.59$; Fig. 3D). We conclude that these data on the RNA level support our results obtained by semiquantitative immunohistochemistry on the protein level. Moreover, these results suggest that at least on the RNA level increased overall Rac expression in prostate cancer results mainly from up-regulation of Rac3 and only to a minor extent from up-regulation of Rac1.

\section{Evaluating Rac3 expression in prostate cancer and its benign counterpart by immunoblotting}

In the next step, we investigated whether increased Rac3 expression in prostate cancer as determined on the RNA level can also be observed on the protein level by immunoblotting. So far, only two Rac3-specific antibodies have been described in the literature (Haataja et al. 1997, Bolis et al. 2003). Of these, one antibody was recently found to cross-react with another yet unknown protein of approximately the same molecular weight as Rac3 (Cho et al. 2005), strongly limiting its applicability. Thus, the only Rac3-specific antibody available so far is the one generated by Dr Ivan de Curtis' group (Bolis et al. 2003). However, this antibody is unsuitable for immunohistochemistry on paraffin-embedded tissues (Dr I de Curtis, personal communication). Therefore, we applied this antibody in the present study to determine Rac3 protein expression by immunoblotting and using frozen tissue samples of the same patients as analyzed for Rac3 expression on the RNA level. Due to the limited amount of available frozen tissue samples, these analyses had to be restricted to patients no. 2, 5, and 6. As shown in Fig. 3E, all prostate carcinomas analyzed exhibited markedly higher Rac3 expression levels than the respective normal counterpart. Increased Rac3 expression in prostate cancer did not result from unequal protein loading as verified by re-incubating the same membrane with an antibody against GAPDH. These results are in line with the results obtained by real-time PCR and therefore suggest that increased Rac expression in prostate cancer, when compared with the respective normal counterpart, results mainly from increased expression of the Rac3 isoform.

\section{Discussion}

There is a great need for new reliable markers, allowing accurate prediction of prostate cancer recurrence, since the markers typically used so far are not sufficiently precise (Ross et al. 2003). In the present study, we investigated the prognostic relevance of Rac expression in human prostate cancer for the first time. We show that Rac protein expression is significantly higher in prostate carcinomas and premalignant HG-PIN lesions than in the corresponding benign secretory epithelial cells. In addition, multivariate analysis, including several factors typically used to predict the prognosis of patients with prostate cancer, suggests that Rac overexpression in prostate cancer relative to the benign epithelial counterpart is a new independent predictor of disease recurrence. Moreover, we provide evidence that increased Rac expression in prostate cancer results mainly from up-regulation of the Rac3 isoform.

In our study, Rac protein expression was mainly investigated by semiquantitative immunohistochemistry, using a monoclonal Rac-specific antibody of which we showed that it recognizes all Rac isoforms, known to be expressed by epithelial cells (i.e. Rac1 and Rac3). We found that overall Rac protein expression levels were significantly higher in prostate cancer than in the respective corresponding benign secretory epithelium $(P<0.001)$. This was supported by the fact that in additional cases, representatively analyzed by real-time PCR, the sum of Rac1 and Rac3 RNA expression levels was also significantly higher in prostate cancer than in the respective benign counterparts $(P=0.018)$. These results indicate that increased Rac expression is a common phenomenon in prostate cancer.

In search for the relative contribution of Rac1 and Rac3 isoforms to increased Rac expression in prostate cancer, we found by isoform-specific real-time PCR that all prostate carcinomas analyzed, exhibited significantly higher Rac3 expression levels (mean, 3.1-fold) than the respective normal counterparts. This 

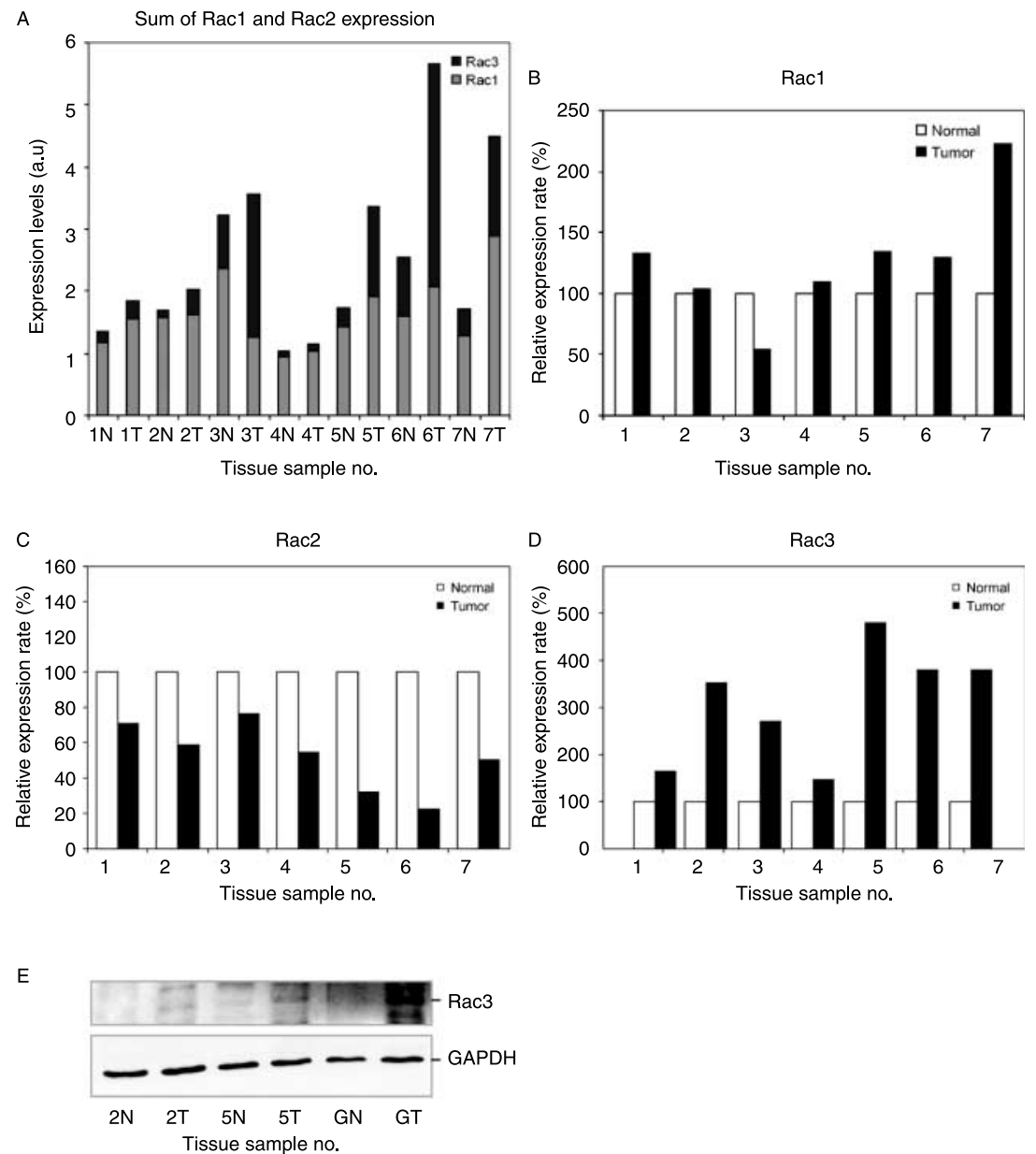

Figure 3 Rac expression levels in prostate cancer and the respective normal counterparts of different patients as determined by realtime PCR and immunoblotting. Histologically verified samples of tumor $(\mathrm{T})$ and corresponding normal tissues $(\mathrm{N})$ were analyzed by Rac1-, Rac2-, and Rac3-specific real-time PCR respectively as well as by immunoblotting with a Rac3-specific antibody. RNA expression levels of Rac1, Rac2, and Rac3 were normalized to the expression level of GAPDH. (A) Overall expression levels of Rac isoforms, known to be expressed by epithelial cells (i.e. sum of Rac1 and Rac3 expression levels; a.u., arbitrary units). (B) Relative expression levels of Rac1 in prostate cancer samples as compared with the respective normal counterparts, the latter of which were defined as $100 \%$. (C) Relative expression levels of Rac2 in prostate cancer samples as compared with the respective normal counterparts, the latter of which were defined as $100 \%$. As Rac2 is known to be specifically expressed by cells of hematopoietic origin, Rac2 expression was used to compare the extent of inflammation in the respective tissue samples. (D) Relative expression levels of Rac3 in prostate cancer samples as compared with the respective normal counterparts, the latter of which were defined as $100 \%$. (E) Rac3 protein expression in prostate cancer samples and the respective normal counterparts as determined by immunoblotting. Due to the limited amount of available frozen tissue samples, these analyses had to be restricted to tissue samples of patients, no. 2, 5, and 6 . Equal protein loading was verified by re-incubationg the same membranes with an antibody against GAPDH. (A)-(E) Results are representative for two independent experiments.

also held true for the protein level as shown by immunoblotting of representative tissue samples of the same patients. In most of the cases examined by real-time PCR, Rac1 expression levels were also higher in tumor tissues than in the corresponding normal tissue samples, but these differences were markedly less pronounced than for Rac3 and probably due to the limited number of cases $(n=7)$, they did not reach statistical significance. As Rac1 is also expressed by inflammatory cells, one might speculate that differences in Rac1 expression as detected by real-time PCR between tumor and normal tissue samples were merely caused by differences in the extent of inflammation. However, this was not the case as verified by histological examination of the respective frozen tissue sections (data not shown) and by Rac2-specific realtime PCR. Interestingly, on average there was a threefold increase in the ratio of Rac3/Rac1 expression levels in all prostate carcinomas when compared with the respective normal counterparts. This suggests that 
increased overall Rac expression in prostate cancer results mainly from up-regulation of Rac 3 and only to a minor extent from up-regulation of Rac1.

Neither the expression nor the role of Rac3 has been investigated in prostate cancer yet, and only very little is known about the role of Rac1 in prostate cancer. Given the known oncogenic/transforming potentials of Rac1 and Rac3 in other cell types (Qiu et al. 1995, Joyce \& Cox 2003, Servitja et al. 2003), one might speculate that Rac signaling plays a major role in prostate carcinogenesis. This is supported by the fact that significantly increased Rac protein expression levels were not only observed in prostate cancer, but also in preneoplastic HG-PIN lesions, suggesting that increased Rac expression occurs early in prostate carcinoma development. We are aware of the fact that increased Rac expression does not necessarily implicate increased Rac activity. Rac proteins are known to cycle between a GDP-bound inactive and a GTP-bound active state (Wennerberg \& Der 2004) and antibodies specific for either of these states are not available so far. Nevertheless, in our cohort, we have recently shown that the Rac-specific activator, Tiam1, is overexpressed in almost all prostate carcinomas (Engers et al. 2006a) and interestingly, overexpression of Rac significantly correlates with strong Tiam1 overexpression (data not shown). This suggests that at least in this cohort increased Rac expression is likely to reflect increased Rac activity. The potential mechanism through which Rac signaling might induce prostate carcinogenesis remains to be determined. Rac stimulates several important signaling pathways, including the p38 mitogen-activated protein kinase, the c-Jun N-terminal Kinase, and the extracellular signal-regulated kinase pathways (Coso et al. 1995, Zhang et al. 1995, Frost et al. 1996), which are known to regulate gene transcription. Therefore, it is conceivable that increased Rac expression might induce transcription of distinct oncogenes and/or inhibit transcription of distinct tumor suppressor genes, which consequently results in oncogenic transformation.

In addition to their effects on transformation, Rac1 and Rac3 have also been implicated in the regulation of migration and invasion of tumor cells (Price \& Collard 2001, Chan et al. 2005), but studies on Rac3 in this respect are very scarce. Interestingly, the effects of Rac1 on migration and invasion in vitro may be either stimulating or inhibitory, depending on the cell-substrate used, the fact as to whether or not the formation of E-cadherin-mediated cell-cell adhesions is prevented, and the cell type studied (Sander et al.
1998, Braga et al. 1999, Engers et al. 2001, Price \& Collard 2001). Given these heterogeneous effects of Rac1 on migration and invasion of epithelial cells in vitro, the effect of increased Rac expression in a given tumor in vivo on DFS might be positive or negative. In prostate cancer, we found that increased Rac expression (relative to the corresponding benign secretory epithelium) is significantly associated with decreased DFS in univariate and most importantly also in multivariate analysis, including several factors typically used to predict the prognosis of patients with prostate cancer. This suggests that in prostate cancer increased Rac expression is an indicator of tumor aggressiveness. In addition, our results suggest that Rac overexpression is a new independent predictor of disease recurrence for patients with prostate cancer and that tumors with higher Rac expression levels than in the corresponding benign secretory epithelium require more aggressive treatment.

The cohort of our study is restricted to 60 patients with R0-resected prostate cancer including 53 patients with appropriate follow-up. Despite its limited size, the strength of this cohort is its restriction to R0-resected tumors, because thus disease recurrence indeed reflects tumor aggressiveness (i.e. the development of metastasis) rather than being merely the result of incomplete surgical excision of the primary tumor. Furthermore, our cohort is representative as evidenced by the fact that well-established prognostic factors, including preoperative PSA level, pT stage, and GS, were also significantly associated with disease recurrence in both univariate and multivariate analysis in our study. Of these, only the preoperative PSA level lost its prognostic effect when Rac overexpression was included into the multivariate analysis. To date, we have evaluated Rac expression only in radical prostatectomy specimens, but if this marker is to be used as part of a pretreatment decisionmaking tool, its predictive power has to be validated in studies on biopsy specimens as well.

In conclusion, the present study suggests that increased Rac protein expression is an early event in prostate cancer development, which appears to result mainly from up-regulation of the Rac3 isoform. Moreover, Rac overexpression in prostate cancer relative to the corresponding benign prostate epithelial cells is an independent predictor of disease recurrence, which in addition to the so far established prognostic factors in prostate cancer, provides valuable information to the clinician in order to optimize the postoperative treatment planning of individual patients. It will be interesting to analyze its predictive 
power in further studies on biopsy specimens as well as in other tumor cell types.

\section{Acknowledgements}

We thank Dr I De Curtis for providing us with the Rac3specific antibody, Dr J G Collard for providing us with the Rac1 and Rac3 constructs, Prof. Dr W A Schulz for sharing some tumor samples with us, and S Khalil, C Collins, S Schneeloch, C Feldhoff, and M Bellack for their excellent technical assistance. Some of the results are part of the medical thesis of A Walter. The authors declare that there is no conflict of interest that would prejudice the impartiality of this scientific work.

\section{References}

Bolis A, Corbetta S, Cioce A \& de Curtis I 2003 Differential distribution of Rac1 and Rac3 GTPases in the developing mouse brain: implications for a role of Rac3 in Purkinje cell differentiation. European Journal of Neuroscience 18 2417-2424.

Braga VM, Del Maschio A, Machesky L \& Dejana E 1999 Regulation of cadherin function by Rho and Rac: modulation by junction maturation and cellular context. Molecular Biology of the Cell 10 9-22.

Carstanjen D, Yamauchi A, Koornneef A, Zang H, Filippi MD, Harris C, Towe J, Atkinson S, Zheng Y, Dinauer MC et al. 2005 Rac 2 regulates neutrophil chemotaxis, superoxide production, and myeloid colony formation through multiple distinct effector pathways. Journal of Immunology 174 4613-4620.

Chan AY, Coniglio SJ, Chuang YY, Michaelson D, Knaus UG, Philips MR \& Symons M 2005 Roles of the Rac1 and Rac3 GTPases in human tumor cell invasion. Oncogene 24 7821-7829.

Cho YJ, Zhang B, Kaartinen V, Haataja L, de Curtis I, Groffen J \& Heisterkamp N 2005 Generation of rac3 null mutant mice: role of Rac3 in Bcr/Abl-caused lymphoblastic leukemia. Molecular and Cellular Biology 25 5777-5785.

Corbetta S, Gualdoni S, Albertinazzi C, Paris S, Croci L, Consalez GG \& de Curtis I 2005 Generation and characterization of Rac3 knockout mice. Molecular and Cellular Biology 25 5763-5776.

Coso OA, Chiariello M, Yu JC, Teramoto H, Crespo P, Xu N, Miki T \& Gutkind JS 1995 The small GTP-binding proteins Rac1 and $\mathrm{Cdc} 42$ regulate the activity of the JNK/SAPK signaling pathway. Cell 81 1137-1146.

Croker BA, Handman E, Hayball JD, Baldwin TM, Voigt V, Cluse LA, Yang FC, Williams DA \& Roberts AW $2002 a$ Rac2-deficient mice display perturbed T-cell distribution and chemotaxis, but only minor abnormalities in $\mathrm{T}(\mathrm{H}) 1$ responses. Immunology and Cell Biology 80 231-240.
Croker BA, Tarlinton DM, Cluse LA, Tuxen AJ, Light A, Yang FC, Williams DA \& Roberts AW 2002b The Rac2 guanosine triphosphatase regulates B lymphocyte antigen receptor responses and chemotaxis and is required for establishment of B-1a and marginal zone B lymphocytes. Journal of Immunology 168 3376-3386.

Didsbury J, Weber RF, Bokoch GM, Evans T \& Snyderman R 1989 Rac, a novel ras-related family of proteins that are botulinum toxin substrates. Journal of Biological Chemistry 264 16378-16382.

Engers R, Springer E, Michiels F, Collard JG \& Gabbert HE 2001 Rac affects invasion of human renal cell carcinomas by up-regulating tissue inhibitor of metalloproteinases (TIMP)-1 and TIMP-2 expression. Journal of Biological Chemistry 276 41889-41897.

Engers R, Mueller M, Walter A, Collard JG, Willers R \& Gabbert HE 2006a Prognostic relevance of Tiam1 protein expression in prostate carcinomas. British Journal of Cancer 95 1081-1086.

Engers R, Springer E, Kehren V, Simic T, Young DA, Beier J, Klotz LO, Clark IM, Sies H \& Gabbert HE $2006 b$ Rac upregulates tissue inhibitor of metalloproteinase-1 expression by redox-dependent activation of extracellular signal-regulated kinase signaling. FEBS Journal 273 4754-4769.

Frost JA, Xu S, Hutchison MR, Marcus S \& Cobb MH 1996 Actions of Rho family small $\mathrm{G}$ proteins and $\mathrm{p} 21$-activated protein kinases on mitogen-activated protein kinase family members. Molecular and Cellular Biology 16 3707-3713.

Gleason DF 1988 Histologic grade, clinical stage, and patient age in prostate cancer. National Cancer Institute Monographs 7 15-18.

Gu Y, Byrne MC, Paranavitana NC, Aronow B, Siefring JE, D'Souza M, Horton HF, Quilliam LA \& Williams DA 2002 Rac2, a hematopoiesis-specific Rho GTPase, specifically regulates mast cell protease gene expression in bone marrow-derived mast cells. Molecular and Cellar Biology 22 7645-7657.

Gu Y, Filippi MD, Cancelas JA, Siefring JE, Williams EP, Jasti AC, Harris CE, Lee AW, Prabhakar R, Atkinson SJ et al. 2003 Hematopoietic cell regulation by Rac1 and Rac2 guanosine triphosphatases. Science 302 445-449.

Haataja L, Groffen J \& Heisterkamp N 1997 Characterization of RAC3, a novel member of the Rho family. Journal of Biological Chemistry 272 20384-20388.

Hordijk PL, ten Klooster JP, van der Kammen RA, Michiels F, Oomen LC \& Collard JG 1997 Inhibition of invasion of epithelial cells by Tiam1-Rac signaling. Science $\mathbf{2 7 8}$ 1464-1466.

Jemal A, Siegel R, Ward E, Murray T, Xu J, Smigal C \& Thun MJ 2006 Cancer statistics, 2006. CA A Cancer Journal for Clinicians 56 106-130. 
Joyce PL \& Cox AD 2003 Rac1 and Rac3 are targets for geranylgeranyltransferase I inhibitor-mediated inhibition of signaling, transformation, and membrane ruffling. Cancer Research 63 7959-7967.

Keely PJ, Westwick JK, Whitehead IP, Der CJ \& Parise LV 1997 Cdc42 and Rac1 induce integrin-mediated cell motility and invasiveness through PI(3)K. Nature 390 632-636.

Kuroda S, Fukata M, Nakagawa M, Fujii K, Nakamura T, Ookubo T, Izawa I, Nagase T, Nomura N, Tani H et al. 1998 Role of IQGAP1, a target of the small GTPases $\mathrm{Cdc} 42$ and Rac1, in regulation of E-cadherin- mediated cell-cell adhesion. Science 281 832-835.

Malliri A, van der Kammen RA, Clark K, van der V, Michiels F \& Collard JG 2002 Mice deficient in the Rac activator Tiam1 are resistant to Ras-induced skin tumours. Nature 417 867-871.

Mira JP, Benard V, Groffen J, Sanders LC \& Knaus UG 2000 Endogenous, hyperactive Rac3 controls proliferation of breast cancer cells by a p21-activated kinase-dependent pathway. PNAS 97 185-189.

Nelson WG, De Marzo AM \& Isaacs WB 2003 Prostate cancer. New England Journal of Medicine 349 366-381.

Olson MF, Ashworth A \& Hall A 1995 An essential role for Rho, Rac, and Cdc42 GTPases in cell cycle progression through G1. Science 269 1270-1272.

Partin AW, Kattan MW, Subong EN, Walsh PC, Wojno KJ, Oesterling JE, Scardino PT \& Pearson JD 1997 Combination of prostate-specific antigen, clinical stage, and Gleason score to predict pathological stage of localized prostate cancer. A multi-institutional update. Journal of the American Medical Association 277 1445-1451.

Price LS \& Collard JG 2001 Regulation of the cytoskeleton by Rho-family GTPases: implications for tumour cell invasion. Seminars in Cancer Biology 11 167-173.

Qiu RG, Chen J, Kirn D, McCormick F \& Symons M 1995 An essential role for Rac in Ras transformation. Nature 374 457-459.
Ridley AJ, Paterson HF, Johnston CL, Diekmann D \& Hall A 1992 The small GTP-binding protein rac regulates growth factor-induced membrane ruffling. Cell 70 401-410.

Ross JS, Jennings TA, Nazeer T, Sheehan CE, Fisher HA, Kauffman RA, Anwar S \& Kallakury BV 2003 Prognostic factors in prostate cancer. American Journal of Clinical Pathology 120 S85-100.

Sander EE, van Delft S, ten Klooster JP, Reid T, van der Kammen RA, Michiels F \& Collard JG 1998 Matrix-dependent Tiam1/Rac signaling in epithelial cells promotes either cell-cell adhesion or cell migration and is regulated by phosphatidylinositol 3-kinase. Journal of Cell Biology 143 1385-1398.

Servitja JM, Marinissen MJ, Sodhi A, Bustelo XR \& Gutkind JS 2003 Rac1 function is required for Src-induced transformation. Evidence of a role for Tiam1 and Vav2 in Rac activation by Src. Journal of Biological Chemistry 278 34339-34346.

Shan D, Chen L, Njardarson JT, Gaul C, Ma X, Danishefsky SJ \& Huang XY 2005 Synthetic analogues of migrastatin that inhibit mammary tumor metastasis in mice. PNAS 102 3772-3776.

Skorski T, Wlodarski P, Daheron L, Salomoni P, NieborowskaSkorska M, Majewski M, Wasik M \& Calabretta B 1998 BCR/ABL-mediated leukemogenesis requires the activity of the small GTP-binding protein Rac. PNAS 95 11858-11862.

Sobin LH \& Wittekind Ch 2002 TNM Classification of Malignant Tumours. Hoboken, New Jersey: John Wiley \& Sons.

Sugihara K, Nakatsuji N, Nakamura K, Nakao K, Hashimoto R, Otani H, Sakagami H, Kondo H, Nozawa S, Aiba A et al. $1998 \mathrm{Rac} 1$ is required for the formation of three germ layers during gastrulation. Oncogene 17 3427-3433.

Wennerberg K \& Der CJ 2004 Rho-family GTPases: it's not only Rac and Rho (and I like it). Journal of Cell Science 117 1301-1312.

Zhang S, Han J, Sells MA, Chernoff J, Knaus UG, Ulevitch RJ \& Bokoch GM 1995 Rho family GTPases regulate p38 mitogen-activated protein kinase through the downstream mediator Pak1. Journal of Biological Chemistry 270 23934-23936. 